\title{
Beating the Odd: 4-year Survival with Primary Cardiac Angiosarcoma
}

\author{
Sarah Louise Picardo ${ }^{1 *}$, Ciara Kelly ${ }^{1}$, Michael Jeffers ${ }^{2}$, Siobhan Nicholson ${ }^{3}$, Orla Buckley ${ }^{4}$, Mary \\ Sheppard $^{5}$, Ian Judson ${ }^{6}$, Alexia Bertuzzi ${ }^{1}$, Janice Walshe ${ }^{1}$
}

${ }^{1}$ Department of Medical Oncology, AMNCH, Tallaght, Dublin, Ireland

${ }^{2}$ Department of Histopathology, AMNCH, Tallaght, Dublin, Ireland

${ }^{3}$ Department of Histopathology, St. James's Hospital, Dublin, Ireland

${ }^{4}$ Department of Radiology, AMNCH, Tallaght, Dublin, Ireland

${ }^{5}$ Department of Histopathology, Royal Brompton Hospital, London, UK

${ }^{6}$ Department of Cancer Pharmacology \& Medical Oncology, Royal Marsden Hospital, London, UK

*Corresponding author: Sarah Louise Picardo, Department of Medical Oncology, AMNCH, Tallaght, Dublin, Ireland,

E-mail: sarahpicardo@gmail.com

Citation: Picardo, S.L., et al. Beating the Odd: 4-year Survival with Primary Cardiac Angiosarcoma. (2016) Int J Cancer Oncol $3(2): 1-4$

\section{Received Date: June 30, 2016, Accepted Date: July 28, 2016, Published Date: August 03, 2016}

\begin{abstract}
A 49-year-old man presented with a 6-week history of exertional dyspnoea. Echocardiogram demonstrated a pericardial effusion. Pericardiocentesis was performed, but fluid cytology was negative for malignancy. Nine months later, repeat echocardiogram showed a right atrial mass and cardiac MRI showed a heterogeneous mass suspicious for cardiac sarcoma. Histology confirmed a cardiac angiosarcoma, deemed inoperable due to cardiac wall infiltration. He commenced weekly paclitaxel treatment and demonstrated a $70 \%$ reduction in cardiac mass on CT after two cycles of therapy. An application for cardiac transplant was refused. His tumour showed no response to two further cycles of paclitaxel and so he was switched to liposomal doxorubicin. This was stopped prematurely due to grade 3 hand-foot skin toxicity. CT scans also demonstrated further disease progression. He was transferred to the Royal Marsden for resection of his primary tumour, with clear margins on resection specimen. He remained disease-free for ten months when liver lesions were identified on staging CT. Biopsy confirmed metastatic disease. He was treated with a tyrosine kinase inhibitor and an mTOR inhibitor but ultimately progressed and therapy was discontinued at patient request. Four months later he represented with symptomatic anaemia. CT showed disease progression with a large hepatic metastasis eroding into omentum. He was treated with further chemotherapy but passed away 51 months after diagnosis.

We discuss the treatment options available for this rare condition. Primary malignant cardiac tumours are rare. Clinical diagnosis is often delayed so patients present with advanced stage disease, usually involving the right atrium, often inoperable at presentation. Cardiac angiosarcoma is particularly aggressive, with median survival of 6-12 months. The benefit of chemotherapy in this setting is usually short-lived; with the regimens most commonly used involving doxorubicin or paclitaxel, although more recent studies have suggested a role for targeted agents such as tyrosine kinase inhibitors or mTOR inhibitors. To our knowledge, this case represents the longest documented survival in adult cardiac angiosarcoma and highlights the need for multidisciplinary care in this rare disease.
\end{abstract}

\section{Case Report}

A 49-year-old man with no previous medical history presented to the emergency department with a 6-week history of exertional dyspnoea. Chest X-ray demonstrated cardiomegaly and echocardiogram demonstrated a large pericardial effusion. The patient underwent pericardiocentesis, draining 1.2 litres of haemorrhagic pericardial fluid. Fluid cytology was negative for malig- 
nancy and fluid culture including TB culture was also negative. Computerised tomography of thorax, abdomen and pelvis (CT TAP) demonstrated small bilateral pleural effusions and mild pericardial thickening, but no obvious malignancy. He was discharged for a follow-up echocardiogram. Nine months later, an echocardiogram demonstrated a right atrial mass (Figure 1(A)). CT and cardiac magnetic resonance imaging (MRI) showed a large heterogeneous mass in the right atrium extending towards the right atrio-ventricular groove, measuring $9.3 \times 5.9 \mathrm{~cm}$ (Figure 1(B) and (C)). Haemorrhage within the mass and patchy enhancement was features suggestive of a cardiac sarcoma. He underwent a coronary angiogram and cardiac biopsy but histology demonstrated normal cardiac myocardium only. Due to high suspicion for malignancy, he proceeded to open cardiac biopsy, which was complicated by intraoperative bleeding. Histology on this occasion confirmed a primary cardiac angiosarcoma, staining positively on immunohistochemistry for CD31, CD34 and WT-1. The tumour was deemed inoperable due to infiltration of the cardiac wall. Staging CT TAP demonstrated no evidence of distant metastases. An application for cardiac transplant was refused.

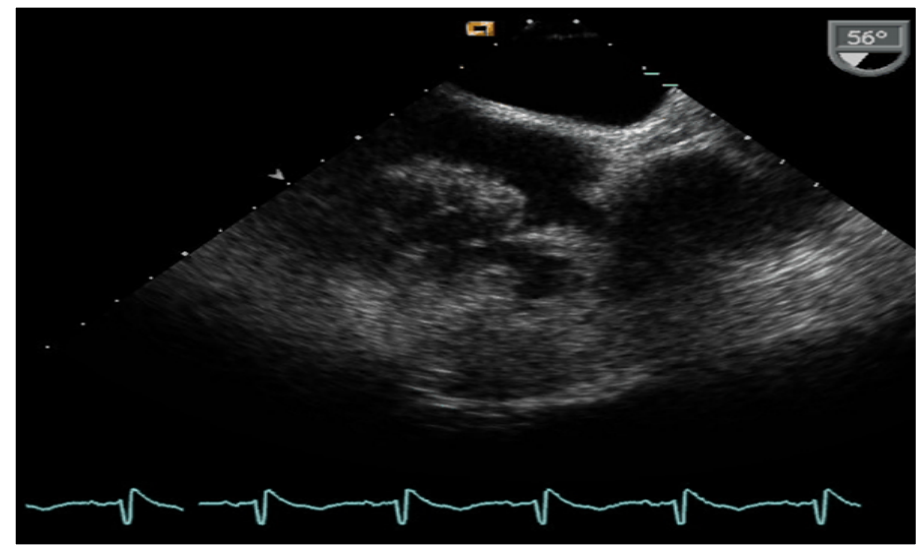

Figure 1: (A) Transoesophageal echocardiogram demonstrates right atrial mass measuring approximately $10 \times 6 \mathrm{~cm}$.

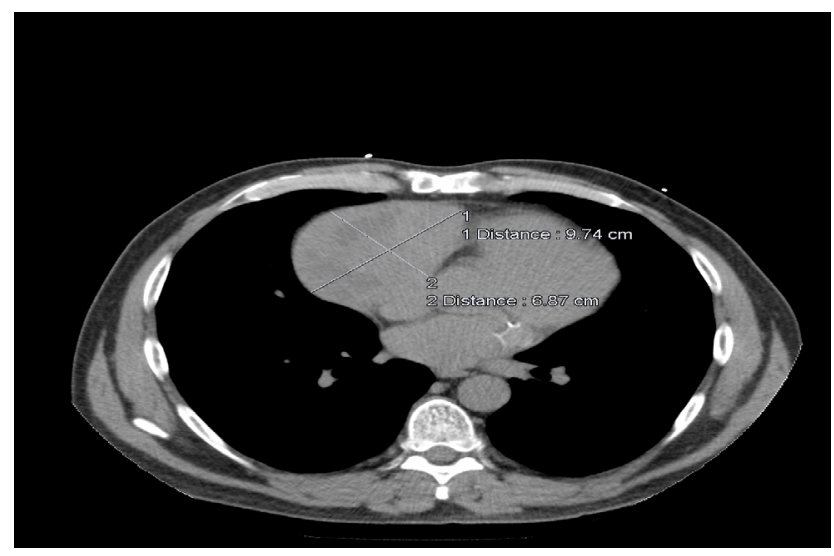

Figure 1: (B) Non contrast CT thorax demonstrates a large mass lesion which is solid with central necrosis in the region of the right atrial wall and pericardium $(9.7 \times 6.8 \mathrm{~cm})$.

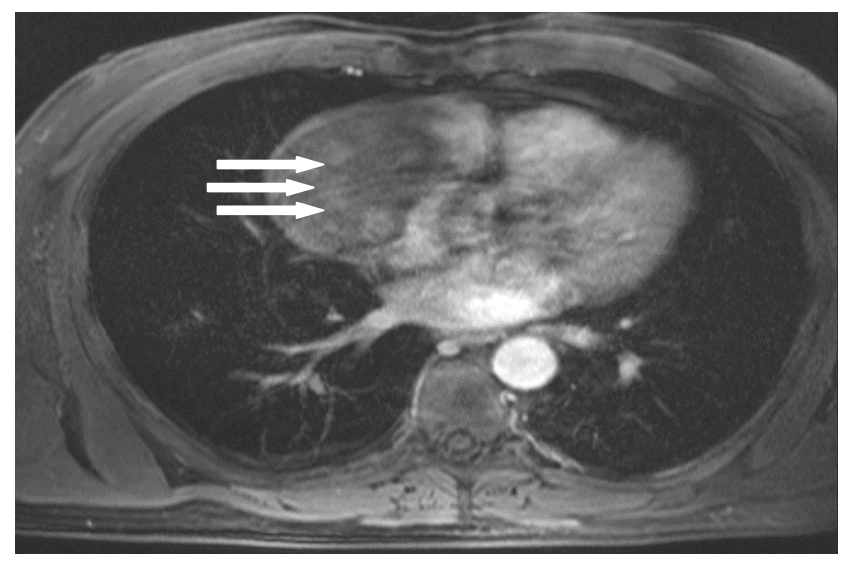

Figure 1: (C) Axial T1 fat saturated MRI post contrast demonstrates heterogenous enhancement of the mass lesion in the right atrium. It is difficult to be sure where the primary origin is of the mass, with differential diagnoses including a pericardial metastases or pericardial mesothelioma. The most common malignant tumour of the heart is sarcoma which tends to be right atrial in origin and this is suspected due to rapid growth and MRI appearance. 
He commenced weekly paclitaxel chemotherapy. He had an excellent response to treatment, with a $70 \%$ reduction in cardiac mass on CT after two cycles of therapy. However, his tumour demonstrated local disease progression after two further cycles of paclitaxel and he was switched to liposomal doxorubicin. This was stopped prematurely due to grade 3 hand-foot skin toxicity and CT scans also demonstrated further local disease progression. Positron emission tomography (PET) scan confirmed local disease only and he was transferred to the Royal Marsden Hospital, London, for resection of his primary tumour and adherent surrounding pericardium, with reconstruction of the right atrium with bovine graft. Resection margins of the excised tumour were clear. Histology confirmed angiosarcoma (Figure 2). He required an intraoperative coronary artery bypass graft due to resection of the right coronary artery. No adjuvant treatment was proposed.

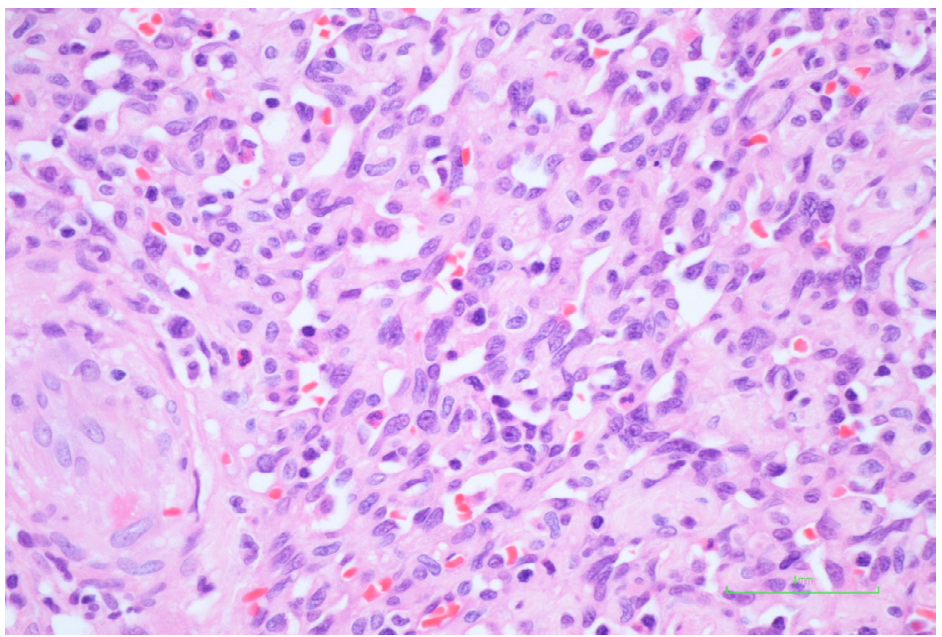

Figure 2: Histology of resection specimen demonstrates tumour composed of interlacing vascular spaces lined by malignant spindle cells, confirming a diagnosis of angiosarcoma.

He remained disease-free for eight months until a staging scan identified two hypoattenuating lesions in the liver suspicious for disease recurrence. A liver biopsy confirmed metastatic disease. He was re-challenged with paclitaxel but he had progressive disease. On the basis of histology, the Tyrosine Kinase Inhibitor (TKI) pazopanib was commenced. After three months, CT again demonstrated disease progression in both liver and mediastinum. As a result, he was switched to gemcitabine and sirolimus. While staging CT demonstrated a decrease in size of hepatic metastases, he tolerated this treatment poorly, developing lower limb cellulitis and severe fatigue. Therapy was discontinued at patient request. Four months later, he represented with symptomatic anaemia and a haemoglobin value of $6.6 \mathrm{~g} / \mathrm{dL}$. CT showed disease progression with ascites and a large hepatic metastasis measuring $14.8 \times 12.1 \mathrm{~cm}$ eroding into the omentum. Ascitic tap showed haemorrhagic ascites and he proceeded to have hepatic artery embolisation, after which his haemoglobin stabilised. Gemcitabine was subsequently re-introduced but liver and mediastinal metastases continued to progress after two months of treatment (Figure 3). He was commenced on single agent doxorubicin. Fifty one months after diagnosis, the patient deteriorated and passed away.
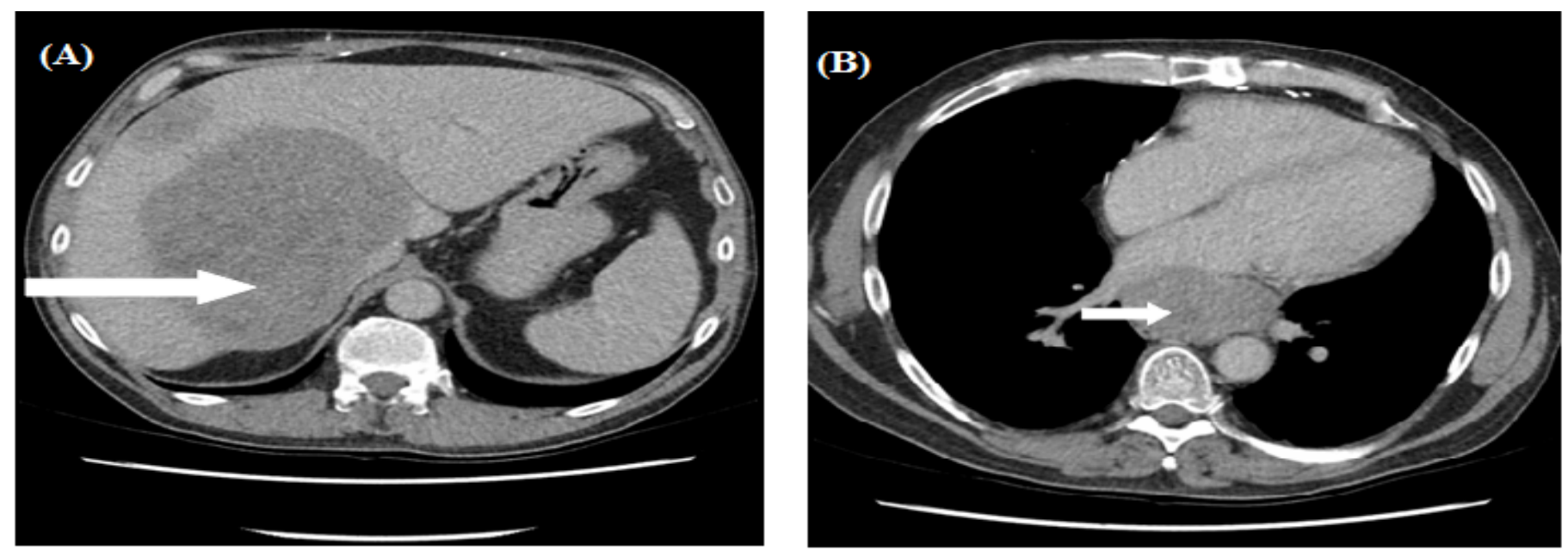

Figure 3: CT thorax and abdomen demonstrates (A) large hepatic metastases and (B) large paraoesophageal posterior thoracic nodal metastasis.

\section{Discussion}

Primary cardiac tumours are extremely rare, with autopsy prevalence estimated at between $0.001 \%$ and $0.03 \%{ }^{[1,2]}$. Seventy-five percent of tumours are benign, with atrial myxomas comprising the majority of these. Twenty-five percent are malignant, of 
which $95 \%$ are sarcomas. The most common histological subtype is angiosarcoma, with a cumulative prevalence of $0.03-0.0001 \%$ at autopsy ${ }^{[3]}$. These tumours, derived from endothelial cells, have been identified in the heart (more often right atrium), aorta or great vessels. Symptoms often occur only at advanced stage of disease, and include dyspnoea on exertion, arrhythmias, and congestive cardiac failure, pericardial effusion causing tamponade, embolic phenomena and systemic symptoms such as fatigue, weight loss and malaise. The male to female ratio is approximately $2: 1$, and they usually occur in young or middle-aged adults ${ }^{[4,5]}$.

The most sensitive diagnostic imaging technique is transoesophageal echocardiography with sensitivity of $97 \%$ while transthoracic echocardiography may not detect right atrial masses ${ }^{[6,7]}$. Further investigations may include coronary angiography, cardiac MRI and staging CT and positron emission tomography (PET) scans ${ }^{[5,8,9]}$. Although many patients present with a pericardial effusion, cytology is often non-diagnostic ${ }^{[7]}$, and up to $50 \%$ of endomyocardial biopsies may be normal despite the presence of malignancy ${ }^{[10]}$. Open cardiac biopsy or frozen sections during surgical resection are recommended as primary methods for obtaining a tissue diagnosis ${ }^{[11]}$. Confirmation of histological diagnosis should be made in a referral centre.

The majority of these tumours are high-grade and poorly differentiated. Pathologic features of angiosarcomas include macroscopically haemorrhagic and often necrotic areas, morphologically compatible with high-grade tumour, with mixed areas of solid growth, spindle-like cells and anatomising channels, frequent mitoses and high Ki67 expression ${ }^{[11]}$. Tissue is positive for vascular markers including CD31, CD34, FLI-1, and WT-1, and can also be focally reactive for p53.

Cardiac angiosarcoma is particularly aggressive in comparison to other sites of angiosarcoma, and has an extremely poor prognosis. Despite potentially curative surgery with adequate margins, the disease is characterised by early recurrence and a median survival of $6-12$ months $^{[5,12]}$. It is usually deemed unresectable at diagnosis due to infiltration through the cardiac wall, large size and/or anatomic location. Additionally, the majority of patients present with distant metastases. If resection is feasible, cardiac wall grafting with a bovine patch is usually required. In cases where the extent of tumour in the right heart requires partial cardiectomy for complete resection, cardiac function may be preserved with a cavopulmonary shunt or Fontan-type operation ${ }^{[13]}$. Occasionally explanation of the heart is required for complete resection ${ }^{[14]}$. Patients who undergo radical resection have improved overall survival compared to those who have a suboptimal resection ${ }^{[15]}$. Cardiac transplant has also been used as part of treatment strategies ${ }^{[16]}$. The role of radiation therapy, either as adjuvant or primary treatment, is limited due to its cardiotoxicity ${ }^{[17,18]}$.

In the case of unresectable or metastatic angiosarcoma, systemic chemotherapy is used to downstage tumours and facilitate surgical resection, or to prolong survival in advanced disease. The role of adjuvant chemotherapy after complete resection is still controversial. Although the classical combination of doxorubicin and ifosfamide is considered the best treatment for soft tissue sarcomas, taxane or doxorubicin based chemotherapy has become the appropriate approach for this rare tumour. This is endorsed by both ASCO and ESMO guidelines ${ }^{[19,20]}$. However, due to the rarity of these tumours, prospective data is sparse.

In a retrospective analysis of 125 cases, single agent doxorubicin or doxorubicin in combination with ifosfamide resulted in progression free survival of 3.7 and 5.4 months respectively ${ }^{[21]}$. Liposomal doxorubicin has also been used in soft tissue sarcomas with similar response rates to doxorubicin $(10 \%$ vs. $9 \%)$ but less cardio toxicity ${ }^{[22]}$.

Taxanes have demonstrated activity against angiosarcomas. Paclitaxel administered with varying schedules to 9 patients with facial or scalp angiosarcomas demonstrated regression of disease in 8 patients ${ }^{[23]}$. In a subsequent phase II study of 32 patients with angiosarcomas, a response rate of $62 \%$ with paclitaxel administered either weekly or three weekly was identified ${ }^{[24]}$. In another phase II trial, 30 patients with unresectable angiosarcomas were administered weekly paclitaxel $\left(80 \mathrm{mg} / \mathrm{m}^{2}\right)$. Median time to progression was 4 months and overall survival 8 months ${ }^{[25]}$. Docetaxel also demonstrates activity in angiosarcoma. In 9 patients with cutaneous angiosarcoma, 2 patients achieved a complete response and 4 achieved partial response ${ }^{[26]}$.

In the context of targeted therapy, recent translational research suggests a possible role for tyrosine kinase inhibitors (TKIs) including sorafenib, and pazopanib, due to changes in the signalling pathways involving VEGF and c-kit identified in these tumours $^{[27]}$. In a Phase II trial of pre-treated metastatic soft tissue sarcomas (STS), patients with angiosarcomas achieved the highest response rates, (RECIST response rate $14 \%$ ) $^{[28,29]}$. Similarly, another phase II trial examining use of sorafenib in angiosarcoma found antitumour activity in patients who had been pre-treated with conventional chemotherapy, with a response rate of $23 \%$ and tumour control rate of $40 \%$, but no activity in those who were chemotherapy-naïve ${ }^{[30]}$. The PALETTE trial, a phase III study, examined pazopanib treatment in 369 patients with pre-treated metastatic STS, including angiosarcoma, and found a PFS of 4.6 months with pazopanib compared with 1.6 months with placebo $^{[31]}$.

Compounds which act as inhibitors of mammalian target of rapamycin (mTOR), a component of the phosphatidyl 3-kinase/ AKT signalling pathway, are currently being investigated as a therapeutic option in treatment of advanced STS following encouraging results in vitro. In a phase III trial, ridaforolimus compared with placebo showed a small but significant increase in progression-free survival in metastatic STS (17.7 weeks vs. 14.6 weeks), however, grade 3 adverse events were higher in the treatment group (64.1\% vs. 25.6\%) $)^{[32,33]}$. Everolimus has shown promising results in multiple tumour types including angiosarcoma ${ }^{[34]}$, while in a multicentre phase II trial, 2 out of 3 angiosarcoma patients enrolled demonstrated stable disease at 16 weeks ${ }^{[35]}$. In a phase II trial, the combination of sirolimus and cyclophosphamide achieved 24 weeks PFS in 10 out of 49 patients, one partial response, median PFS of 3.4 months and overall survival of 9.9 months $^{[36]}$.

\section{Conclusion}

In conclusion, there is no consensus on the optimal sequence of treatment in advanced angiosarcoma. To our knowledge, this case represents the longest reported survival in adult cardiac angiosarcoma and highlights the role of a multidisciplinary approach including both surgical and chemotherapeutic treatments in treatment of this rare disease, suggesting that prolonged overall 
survival may be possible with an aggressive surgical approach and newer therapeutic agents.

\section{References}

1. Lam, K.Y., Dickens, P., Chan, A.C. Tumors of the heart. A 20-year experience with a review of 12,485 consecutive autopsies. (1993) Arch Pathol Lab Med 117(10): 1027-1031.

2. Patel, J., Sheppard, M.N. Pathological study of primary cardiac and pericardial tumours in a specialist UK Centre: surgical and autopsy series. (2010) Cardiovasc Pathol 19(6): 343-352.

3. Riberi, A., Gariboldi, V., Grisoli, D., et al. [Cardiac tumors]. (2010) Rev Pneumol Clin 66(1): 95-103.

4. Randhawa, J. S., Budd, G.T., Randhawa, M., et al. Primary Cardiac Sarcoma: 25-Year Cleveland Clinic Experience. (2014) Am J Clin Oncol [Epub ahead of print].

5. Centofanti, P., Di Rosa, E., Deorsola, L., et al. Primary cardiac tumors: early and late results of surgical treatment in 91 patients. (1999) Ann Thorac Surg 68(4): 1236-1241.

6. Meng, Q., Lai, H., Lima, J., et al. Echocardiographic and pathologic characteristics of primary cardiac tumors: a study of 149 cases. (2002) Int J Cardiol 84(1): 69-75.

7. Riles, E., Gupta, S., et al. Primary cardiac angiosarcoma: A diagnostic challenge in a young man with recurrent pericardial effusions. (2012) Exp Clin Cardiol 17(1): 39-42.

8. Jain, A., Simon, S., Elangovan, I., et al. (18)F-fluoro-deoxyglucose positron emission tomography-computed tomography in initial assessment and diagnosis of right atrial angiosarcoma with widespread visceral metastases: A rare case report and review of the literature. (2015) Indian J Nucl Med 30(1): 51-54.

9. Hoey, E.T., Shahid, M., Ganeshan, A., et al. MRI assessment of cardiac tumours: part 2, spectrum of appearances of histologically malignant lesions and tumour mimics. (2014) Quant Imaging Med Surg 4(6): 489-497.

10. Rettmar, K., Stierle, U., Sheikhzadeh, A., et al. Primary angiosarcoma of the heart. Report of a case and review of the literature. (1993) Jpn Heart J 34(5): 667-683.

11. Ge, Y., Ro, J.Y., Kim, D., et al. Clinicopathologic and immunohistochemical characteristics of adult primary cardiac angiosarcomas: analysis of 10 cases. (2011) Ann Diagn Pathol 15(4): 262-267.

12. Truong, P. T., Jones, S.O., Martens, B., et al. Treatment and outcomes in adult patients with primary cardiac sarcoma: the British Columbia Cancer Agency experience. (2009) Ann Surg Oncol 16(12): 3358-3365.

13. Hoffmeier, A., Deiters, S., Schmidt, C., et al. Radical resection of cardiac sarcoma. (2004) Thorac Cardiovasc Surg 52(2): 77-81.

14. Hoffmeier,A., Scheld, H.H., Tjan, T.D., et al. Ex situ resection of primary cardiac tumors. (2003) Thorac Cardiovasc Surg 51(2): 99-101.

15. Pacini, D., Careddu, L., Pantaleo, A., et al. Primary malignant tumors of the heart: Outcomes of the surgical treatment. (2015) Asian Cardiovasc Thorac Ann 23(6): 645-651.

16. Baay, P., Karwande, S.V., Kushner, J.P., et al. Successful treatment of a cardiac angiosarcoma with combined modality therapy. (1994) J Heart Lung Transplant 13(5): 923-925.

17. Franceschini, D., Scotti, V., Simontacchi, G., et al. Application of helical tomotherapy for the treatment of a right atrium angiosarcoma: a case report. (2013) Tumori 99(5): e233-e236.

18. Slepicka, C., Durci, M. Cardiac angiosarcoma treated with resection and adjuvant radiation therapy. (2012) J La State Med Soc 164(2): $92-93$. 19. Scurr, M., Judson, I. Neoadjuvant and adjuvant therapy for extremity soft tissue sarcomas. (2005) Hematol Oncol Clin North Am 19(3): 489500 .

20. Casali, P.G., Jost, L., Sleijfer, S., et al. Soft tissue sarcomas: ESMO clinical recommendations for diagnosis, treatment and follow-up. (2008) Ann Oncol 19(Suppl 2): 89-93.

21. Fury, M.G., Antonescu, C.R., Van Zee, K.J., et al. A 14-year retrospective review of angiosarcoma: clinical characteristics, prognostic factors, and treatment outcomes with surgery and chemotherapy. (2005) Cancer J 11(3): 241-247.

22. Judson, I., Radford, J.A., Harris, M., et al. Randomised phase II trial of pegylated liposomal doxorubicin (DOXIL/CAELYX) versus doxorubicin in the treatment of advanced or metastatic soft tissue sarcoma: a study by the EORTC Soft Tissue and Bone Sarcoma Group. (2001) Eur J Cancer 37(7): 870-877.

23. Fata, F., O'Reilly, E., Ilson, D., et al. Paclitaxel in the treatment of patients with angiosarcoma of the scalp or face. (1999) Cancer 86(10): 2034-2037.

24. Schlemmer, M., Reichardt, P., Verweij, J., et al. Paclitaxel in patients with advanced angiosarcomas of soft tissue: a retrospective study of the EORTC soft tissue and bone sarcoma group. (2008) Eur J Cancer 44(16): 2433-2436.

25. Penel, N., Bui, B.N., Bay, J.O., et al. Phase II trial of weekly paclitaxel for unresectable angiosarcoma: the ANGIOTAX Study. (2008) J Clin Oncol 26(32): 5269-5274.

26. Nagano, T., Yamada, Y., Ikeda, T., et al. Docetaxel: a therapeutic option in the treatment of cutaneous angiosarcoma: report of 9 patients. (2007) Cancer 110(3): 648-651.

27. Sleijfer, S., Ray-Coquard, I., Papai, Z., et al. Pazopanib, a multikinase angiogenesis inhibitor, in patients with relapsed or refractory advanced soft tissue sarcoma: a phase II study from the European organisation for research and treatment of cancer-soft tissue and bone sarcoma group (EORTC study 62043). (2009) J Clin Oncol 27(19): 3126-3132.

28. Maki, R.G., D’Adamo, D.R., Keohan, M.L., et al. Phase II study of sorafenib in patients with metastatic or recurrent sarcomas. (2009) J Clin Oncol 27(19): 3133-3140.

29. Santoro, A., Comandone, A., Basso, U., et al. Phase II prospective study with sorafenib in advanced soft tissue sarcomas after anthracycline-based therapy. (2013) Ann Oncol 24(4): 1093-1098.

30. Ray-Coquard, I., Italiano, A., Bompas, E., et al. Sorafenib for patients with advanced angiosarcoma: a phase II Trial from the French Sarcoma Group (GSF/GETO). (2012) Oncologist 17(2): 260-266.

31. van der Graaf, W.T., Blay, J.Y., Chawla, S.P., et al. Pazopanib for metastatic soft-tissue sarcoma (PALETTE): a randomised, double-blind, placebo-controlled phase 3 trial. (2012) Lancet 379(9829): 1879-1886.

32. Chawla, S.P., Staddon, A.P., Baker, L.H., et al. Phase II study of the mammalian target of rapamycin inhibitor ridaforolimus in patients with 
advanced bone and soft tissue sarcomas. (2012) J Clin Oncol 30(1): 78-84.

33. Demetri, G.D., Chawla, S.P., Ray-Coquard, I., et al. Results of an international randomized phase III trial of the mammalian target of rapamycin inhibitor ridaforolimus versus placebo to control metastatic sarcomas in patients after benefit from prior chemotherapy. (2013) J Clin Oncol 31(19): 2485-2492.

34. Campone, M., Levy, V., Bourbouloux, E., et al. Safety and pharmacokinetics of paclitaxel and the oral mTOR inhibitor everolimus in advanced solid tumours. (2009) Br J Cancer 100(2): 315-321.

35. Yoo, C., Lee, J., Rha, S.Y., et al. Multicenter phase II study of everolimus in patients with metastatic or recurrent bone and soft-tissue sarcomas after failure of anthracycline and ifosfamide. (2013) Invest New Drugs 31(6): 1602-1608.

36. Schuetze, S.M., Zhao, L., Chugh, R., et al. Results of a phase II study of sirolimus and cyclophosphamide in patients with advanced sarcoma. (2012) Eur J Cancer 48(9): 1347-1353. 